\title{
Polymorphism and Uniparental Inheritance of Mitochondrial DNA in Physarum polycephalum
}

\author{
By S. KAWANO, ${ }^{*}$ R. W. ANDERSON,${ }^{2}$ T. NANBA ${ }^{1}$ AND T. KUROIWA ${ }^{1}$ \\ ${ }^{1}$ Department of Cell Biology, National Institute for Basic Biology, 38 Myodaijicho, \\ Okazaki 444, Japan \\ ${ }^{2}$ Department of Genetics, University of Sheffield, Sheffield S10 2TN, UK
}

(Received 10 April 1987; revised 22 June 1987)

\begin{abstract}
Restriction endonuclease analysis was done on mitochondrial DNA (mtDNA) from 19 plasmodial strains of Physarum polycephalum. The extent of mtDNA variation among these strains was high in comparison with other organisms, and provides a useful source of cytoplasmic genetic markers. The strains were classified into seven groups according to their mtDNA types. Although plasmodia of $P$. polycephalum are diploid, formed by fusion of amoebal isogametes, each of the 19 plasmodia possessed mtDNA of only a single type. The transmission pattern of mtDNA during plasmodium formation was studied by mating pairs of amoebal strains that contained mtDNA of different types. Transmission was uniparental; the plasmodia that were formed carried mtDNA with the restriction pattern of only one of the two parental types. Since diploid zygotes develop into plasmodia by repeated mitotic cycles in the absence of cell division, it is clear that this uniparental transmission of mtDNA does not depend upon random partitioning either of mitochondria or of mtDNA molecules during cell division.
\end{abstract}

\section{INTRODUCTION}

Mitochondrial genes are transmitted to progeny predominantly or entirely by only one parent in a number of different organisms. In oogamous species, this uniparental inheritance has usually been attributed to failure of mitochondria from the male gamete to enter the egg, or to the presence in the male gamete of comparatively few organelles (for reviews see Sager, 1972; Birky, 1978). However, these cellular mechanisms cannot explain the production of uniparental, homoplasmic zygotes in species such as yeasts, which do not have differentiated gametes; in such isogamous species, uniparental inheritance is thought to be due at least partly to random partitioning of mitochondria during cell division (for reviews see Birky, 1978; Birky et al., 1982).

The life cycle of the acellular slime mould Physarum polycephalum includes two distinct vegetative forms: the uninucleate amoeba and the multinucleate, syncytial plasmodium. Haploid amoebae act as isogametes, fusing in pairs of different mating type to form diploid zygotes which develop into macroscopic, diploid plasmodia by repeated mitotic cycles in the absence of cell division (reviewed by Dee, 1982). Thus, the random partitioning of mitochondria by cell division does not occur during plasmodium formation. It was of interest to determine whether a heteroplasmic zygote produced by mating would develop into a homoplasmic plasmodium in spite of the absence of such random partitioning.

The inheritance of mitochondrial genes in Physarum has been difficult to study because easily scored cytoplasmic mutants have not been available. In the present work, restriction endonuclease digestion has been used both to study the degree of mtDNA polymorphism among a series of $P$. polycephalum strains, and to determine the pattern of transmission of mtDNA during the process of plasmodium formation.

Abbreviation: mtDNA, mitochondrial DNA.

0001-4136 (C) 1987 SGM 
Table 1. Strains used

(a) Plasmodial strains

Designation

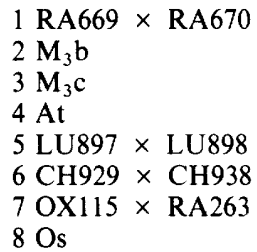

$9 \mathrm{~F} \times$

10 PPO-1

$11 \mathrm{Cb}$

12 To

13 Ho

14 DP74 $\times$ DP75

$15 \mathrm{Nc}$

$16 \mathrm{Ng}$

$17 \mathrm{Hi}$

18 DP12 $\times$ DP13

19 DP89 $\times$ DP90

(b) Amoebal strains

\begin{tabular}{|c|c|c|c|}
\hline Designation & Genc & otype & Reference or origin \\
\hline a & mat $A 1$ & matBI & Dee $(1960)$ \\
\hline $\mathrm{i}$ & $\operatorname{mat} A 2$ & matB4 & Dee $(1960)$ \\
\hline CH929 & matAl & matB3 & Anderson \& Truitt (1983) \\
\hline CH938 & mat $A 4$ & matB2 & Kawano et al. (1987) \\
\hline DP12 & mat A5 & mat $B 10$ & \\
\hline DP13 & mat A6 & matBII & \\
\hline DP74 & matA12 & matB5 & \\
\hline DP75 & matA11 & matB6 & Kirouac-Brunet et al. (1981) \\
\hline DP89 & matA15 & mat $B 12$ & \\
\hline DP90 & mat $A 16$ & matB13 & \\
\hline LU853 & mat $A 1$ & matBI & Cooke (1974) \\
\hline LU887 & mat $A 2$ & matBI & Anderson (1977) \\
\hline LU897 & mat $A I$ & matBI & Anderson (1976) \\
\hline LU898 & mat $A 2$ & matBI & LU853 $\times$ LU887 \\
\hline $\mathrm{OX} 110$ & mat $A 2$ & matBI & \\
\hline OX115 & mat $A 3$ & matBI & Kawano et al. (1987) \\
\hline OX118 & mat $A 3$ & matB3 & \\
\hline RA263 & mat $A 2$ & matB3 & OX110 $\times$ OX118 \\
\hline RA669 & mat $A 1$ & matB4 & $a \times i$ \\
\hline RA670 & mat $A 2$ & matBI & $a \times j$ \\
\hline
\end{tabular}

Reference or origin

Mohberg \& Babcock (1982)

Mohberg \& Babcock (1982)

ATCC 44912

N. Kamiya, Osaka University

Wakasugi \& Ohta (1973)

Kawano et al. (1982)

F. Sawada, National Institute for Radiological Sciences

A. Nishi, Toyama Medical \& Pharmacological University

Y. Kobatake, Hokkaido University

North Carolina biological supply house

S. Hatano, Nagoya University

E. Kamitsubo, Hitotsubashi University

\section{METHODS}

Strains. Principal plasmodial and amoebal strains are listed in Table 1. Amoebae were maintained at $29 \cdot 5^{\circ} \mathrm{C}$ in monoxenic culture with Escherichia coli on SM-1 plates, as described by Kawano et al. (1987). Plasmodia were cultured at $26^{\circ} \mathrm{C}$ on MEA (malt extract agar; Kawano et al., 1987) and microplasmodia were cultured as described by Daniel \& Baldwin (1964). Sporulation was induced on MEA plates, essentially as detailed by Wheals (1970). Germination of spores and cloning of amoebal progeny were done according to the methods of Anderson (1979) but using SM-1 instead of liver infusion agar. Stocks of amoebal strains were maintained as desiccated cysts on silica gel granules (Anderson et al., 1983).

Generation of plasmodia and mating-type analysis. To obtain plasmodia by mating, $10^{5}$ amoebae of each of two strains carrying unlike mat $A$ and $m a t B$ alleles were mixed at $26^{\circ} \mathrm{C}$ in 'concentrated' live $E$. coli suspension and plated as described by Youngman et al. (1981). Following mating, small agar blocks carrying plasmodia were cut from the mating plates and transferred to MEA for further growth. These plasmodia were confirmed to result from mating by analysis of their progeny for mat $A$ and $m a t B$ alleles. Such analyses were done essentially as described by Youngman et al. (1979). 
Table 2. Classification of plasmodia for mtDNA types and mating-type alleles

\begin{tabular}{|c|c|c|c|c|c|}
\hline \multirow[b]{3}{*}{ Strain } & \multirow{3}{*}{$\overbrace{\text { Mating type }}^{\text {Nucleus }}$} & \multicolumn{4}{|c|}{ Mitochondria } \\
\hline & & \multirow[b]{2}{*}{ Genome size } & \multicolumn{2}{|c|}{$\begin{array}{l}\text { Percentage differential } \\
\text { restriction fragments }\end{array}$} & \multirow[b]{2}{*}{ mtDNA type } \\
\hline & & & $(a)^{*}$ & $(b) \dagger$ & \\
\hline $\begin{array}{l}\text { RA669 } \times \text { RA670, } \\
\text { M }_{3} b, M_{3} \mathrm{c}, \text { At, } \\
\text { LU897 } \times \text { LU898, } \\
\text { CH929 } \times \text { CH938, } \\
\text { OX115 } \times \text { RA263 }\end{array}$ & $\begin{array}{l}\text { mat } A I-4 \\
\text { mat } B I-4\end{array}$ & $60 \cdot 1 \pm 4 \cdot 3$ & - & 25.9 & M (McArdle) \\
\hline $\begin{array}{l}\text { Os, F } \times \text { J, } \\
\text { PPO-1, Cb, } \\
\text { To, Ho, }\end{array}$ & Unknown & $68.2 \pm 8.8$ & $43 \cdot 2$ & $44 \cdot 9$ & O (Osaka) \\
\hline $\mathrm{DP} 12 \times \mathrm{DP} 13$ & $\begin{array}{l}\operatorname{mat} A 5,6 \\
\operatorname{mat} B 10,11\end{array}$ & $64 \cdot 7 \pm 3 \cdot 8$ & $37 \cdot 6$ & $39 \cdot 2$ & I (Iowa) \\
\hline DP89 $\times$ DP90 & $\begin{array}{l}\operatorname{mat} A 15,16 \\
\operatorname{mat} B 12,13\end{array}$ & $61 \cdot 1 \pm 7 \cdot 2$ & $18 \cdot 8$ & $15 \cdot 9$ & W (Wisconsin) \\
\hline $\begin{array}{l}\mathrm{DP} 74 \times \mathrm{DP} 75 \\
\mathrm{Nc}\end{array}$ & $\begin{array}{c}\text { mat } A 11,12 \\
\text { matB5,6 }\end{array}$ & $62 \cdot 1 \pm 3.6$ & $25 \cdot 9$ & - & B (Boreal) \\
\hline $\mathrm{Ng}$ & $\begin{array}{c}\text { mat } A 11,12 \\
\text { mat } B 5,6\end{array}$ & $62 \cdot 7 \pm 5 \cdot 7$ & $27 \cdot 0$ & $17 \cdot 1$ & N (Nagoya) \\
\hline $\mathrm{Hi}$ & $\begin{array}{l}\text { mat } A 11,12 \\
\text { mat } B 5,6\end{array}$ & $63.4 \pm 4.5$ & $30 \cdot 7$ & $16 \cdot 1$ & H (Hitotsubashi) \\
\hline & $\begin{array}{l}\text { Comparison } \\
\text { Comparison } \\
\text { Not mat } A 1,2,2\end{array}$ & $\begin{array}{l}\text {-type. } \\
\text {-type. } \\
11,12,15,16\end{array}$ & 2 & 2,13 & \\
\hline
\end{tabular}

Restriction endonuclease analysis of $\mathrm{mt} D N A$. Mitochondria were isolated from microplasmodia or macroplasmodia of each strain as described by Kawano et al. (1983). mtDNA was isolated from isolated mitochondria by centrifugation in Hoechst $33258 / \mathrm{CsCl}$ density gradients according to Hudspeth et al. (1980). Restriction endonucleases were obtained from New England Biolabs and the Takara Shuzo Co. Digests were done in buffers recommended by the manufacturers. Electrophoresis and photography of $1-2 \%(w / v)$ agarose slab gels were as described by Kawano et al. (1983). Percentage differential restriction fragments $\left(p_{i j}\right)$ were calculated by the formula $p_{i j}=100\left(d_{i j} / n_{i}+n_{j}\right)$, where $n_{i}$ and $n_{j}$ are the total fragment numbers observed in the restriction patterns of the $i$ th and $j$ th miDNA types and $d_{i j}$ is the number of differential fragments found between the two types as described by Kaneko et al. (1986). This analysis was done by using the data of six-base-pair-recognizing restriction endonucleases (EcoRI, EcoRV, HindIII, HpaI and $X b a \mathrm{I}$ ).

\section{RESULTS AND DISCUSSION}

Polymorphism of mtDNA revealed by restriction endonuclease analysis

mtDNA isolated from microplasmodia of 19 strains was digested with six restriction endonucleases: Bst NI, EcoRI, EcoRV, HindIII, HpaI and XbaI. Restriction fragment patterns sometimes deviated from normal stoichiometry; in particular, restriction fragments from the terminal regions of mtDNA molecules tended to be seen as faint and diffuse bands (Fig. 1). As reported by Kawano et al. (1982), this phenomenon is attributable to the use of broken linear mtDNA as starting material. Partial breakage of mtDNA is generally observed during isolation of mtDNA from fungi (Sanders et al., 1975) and does not prevent the estimation of mitochondrial genome size or the detection of mtDNA variation. The mitochondrial genome size estimated from the total molecular masses of the restriction fragments differed among the strains (60.1-68.2 kb; see Table 2), but the values were all close to the genome size of $68.2 \mathrm{~kb}$ found for strain PPO-1 by Kawano et al. (1982). As shown in this earlier study, this implies that the mtDNA within each individual strain was of only a single type.

Fig. 1(a) shows the HindIII restriction fragment patterns of mtDNA from the 19 strains. 
(a)

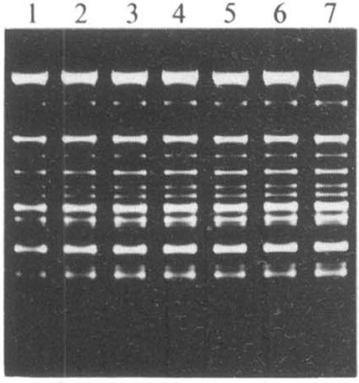

(b)

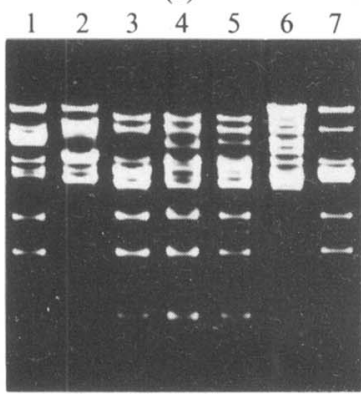

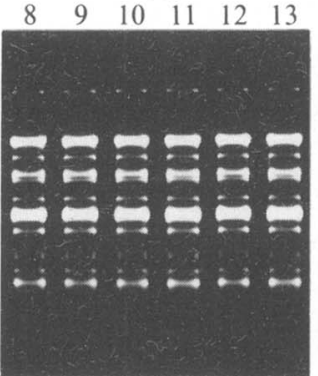

(c)

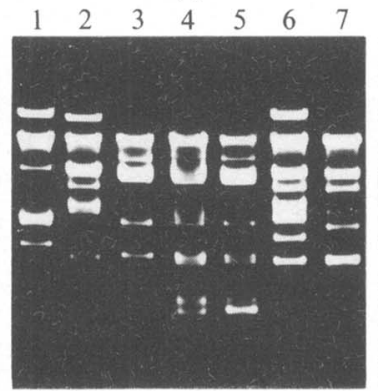

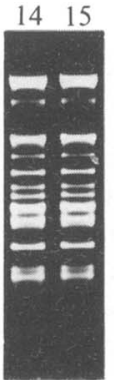

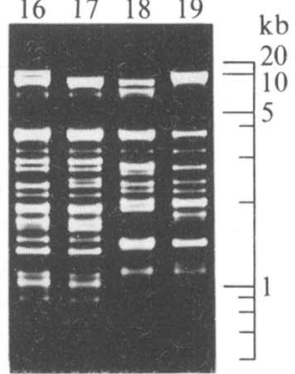

(d)

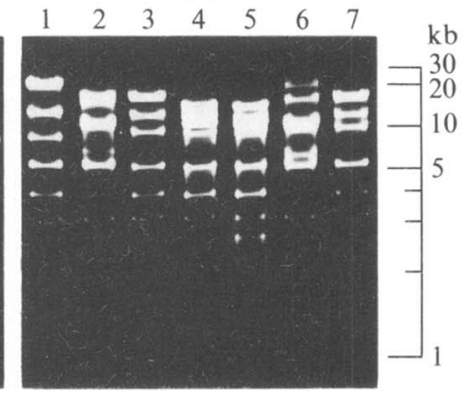

Fig. 1. (a) HindIII restriction patterns of mtDNA from the 19 plasmodial strains. The lanes are numbered in accordance with Table $1(a) .(b)-(d)$, Restriction patterns of the seven mtDNA types with $B s t$ NI (b), EcoRV (c), XbaI $(d)$. The lanes are numbered as follows: 1, M-type mtDNA; 2, O-type; 3, Btype; 4, N-type; 5, H-type; 6, I-type; 7, W-type.

Seven distinct types of mtDNA were detected. Fig. $1(b-d)$ shows representative restriction fragment patterns of these seven distinct mtDNA types digested with BstNI, EcoR V anj $X b a \mathrm{I}$. The digestion patterns showed conservation of certain restriction fragments between the different mtDNA types, but most fragments were unique to each type. Digestions with EcoRI and $\mathrm{HpaI}$ (data not shown) also confirmed the existence of seven groups. The detailed pattern of methylation sites in $P$. polycephalum $\mathrm{mtDNA}$ is unknown, although the involvement of cytosine is clearly established (Kawano et al., 1983); since Bst NI, EcoRI, EcoRV and HpaI are not affected by methylation of cytosines, it is clear that the different restriction patterns were largely based upon differences in nucleotide sequence rather than on differential methylation.

To quantify simply the variation between different mtDNA types, percentage differential restriction fragments, $p_{i j}$, were calculated; these show the frequency of differential restriction fragments observed in the pairwise comparison of the different mtDNA types (Table 2). The mean value of $p_{i j}$ was $28.5 \%(\mathrm{sD}=10 \cdot 2 \%$ ), significantly higher than the percentage differential restriction fragments of animal mtDNA, which range from $2.4 \%$ in humans to $7.8 \%$ in chimpanzees. These values were calculated from the data of Cann et al. (1984) and Ferris et al. (1981), respectively. Strain and specific variations in mtDNAs have also been observed in many fungi, and variation here is generally greater than among animal mtDNAs. For example, in Achlya heterosexualis (Shumard et al., 1986), the percentage differential restriction fragments were calculated to be $18 \cdot 0 \%$.

It is known that polymorphism of animal mtDNA mostly results from single base substitutions (Castora et al., 1980), but it has been postulated that variation in fungi is the result of insertions into or deletions from basically similar genomes in which most restriction endonuclease sites are conserved (Turner et al., 1982). The mitochondrial genome size heterogeneity observed among $P$. polycephalum strains suggests that variations of restriction fragment patterns may in part be caused by such insertions and deletions. Nevertheless, the basis of genome size heterogeneity and nucleotide diversity in $P$. polycephalum $\mathrm{mtDNA}$ is not yet 
certain. The high degree of variability defined in these studies makes it clear that polymorphism of mtDNA is a potentially rich source of cytoplasmic genetic markers in this organism, which has previously lacked any well-defined cytoplasmic mutants.

\section{Origins of the different $m t D N A$ types}

The existence of seven distinct types of mtDNA among the 19 strains studied suggested that each mtDNA type might correspond to a different natural plasmodial isolate of $P$. polycephalum. Some support for this view came from the known common origins of certain strains; in many cases, however, the original isolate was unknown, and information was therefore obtained from tests of mating type. Each natural plasmodial isolate of $P$. polycephalum appears to be characterized by two unique alleles of each of the multiallelic mating-type loci mat $A$ and $m a t B$ (Kirouac-Brunet et al., 1981), so that mating-type alleles are good nuclear gene markers to show the particular isolate from which any strain has been derived.

$\mathrm{M}_{3} \mathrm{~b}$ and $\mathrm{M}_{3} \mathrm{c}$ are sublines of the same isolate, Wis 1, collected by M. Backus about 1947 (Mohberg \& Babcock, 1982); RA669 and RA670 are second-generation meiotic progeny derived from the same isolate. $M_{3} b, M_{3} c$ and RA669 $\times$ RA670 all carried mtDNA of the same type, designated M-type (Table 2). The plasmodium designated At also carried M-type mtDNA, and analysis of its amoebal progeny showed that the mating-type alleles characteristic of the Wis 1 isolate were present: matA1, matA2, matBI and matB4. It thus appears that the At plasmodium, which was obtained from the American Type Culture Collection, was of Wis 1 origin.

Three further plasmodial strains carried M-type mtDNA. These plasmodia were all generated by crosses between pairs of amoebal strains that were largely isogenic with the Colonia isolate, although the strains carried mating-type alleles derived from the Wis 1 and Indiana isolates (Adler \& Holt, 1974; Cooke \& Dee, 1975). The Colonia and Wis 1 isolates are genetically similar in several ways and it has been suggested that Colonia is probably derived from Wis 1 (Honey et al., 1979; see also Kawano et al., 1987). The fact that Colonia-isogenic strains possess M-type mtDNA is consistent with this hypothesis.

The six plasmodial strains possessing O-type mtDNA were all obtained from Japanese laboratories. These plasmodia carried identical mat $A$ and mat $B$ alleles, which were different from those carried by any of the other plasmodia or by any of the mating-type tester strains available to us: mat $A 1,2,3,4,5,6,11,12,15,16$, and matB1, 2, 3, 4, 5, 6, 10, 11, 12, 13.

The I and W mtDNA types were each represented by a single plasmodium with unique mating-type alleles. DP12 $\times$ DP13 was derived from progeny of the plasmodial isolate PpII (Collins, 1975), and DP89 $\times$ DP90 was derived from progeny of the Wis 2 isolate (KirouacBrunet et al., 1981).

Two plasmodia possessed B-type mtDNA: Nc was obtained from the North Carolina biological supply house, and DP74 $\times$ DP75 was generated by mating amoebal progeny of a plasmodium from the Boreal biological supply house. On the basis of mating-type analyses, Kirouac-Brunet et al. (1981) suggested that the Boreal, North Carolina and Turtox supply houses were all culturing the same plasmodium, originally known as the Turtox isolate. Our own mating-type analyses confirm that $\mathrm{Nc}$ carries the same alleles as DP74 $\times$ DP75: matAll and mat $A 12$, matB5 and matB6.

Although $\mathrm{Ng}$ and $\mathrm{Hi}$ each carried a unique mtDNA type, mating-type analyses showed that both these plasmodia carried the same mat $A$ and mat $B$ alleles as $\mathrm{Nc}$ and DP74 $\times$ DP75. Calculation of percentage differential restriction fragments showed that the three mtDNA types involved ( $\mathrm{B}, \mathrm{N}$ and $\mathrm{H}$ ) were not closely related, and thus did not represent minor divergence from an ancestral sequence in the original wild isolate. Unless that isolate carried three different mtDNA species which have since become segregated into different sublines, it would appear that exchanges of cytoplasm must have occurred between isolates. However, it is difficult to see how such exchanges would take place. Mating between amoebae of different isolates occurs readily, but the plasmodial products of such matings contain only a single mat $A$ and mat $B$ allele from each parent. A second possible mechanism of cytoplasmic exchange would be plasmodial fusion, but the large number of incompatibility genes controlling somatic fusions between 
(a)

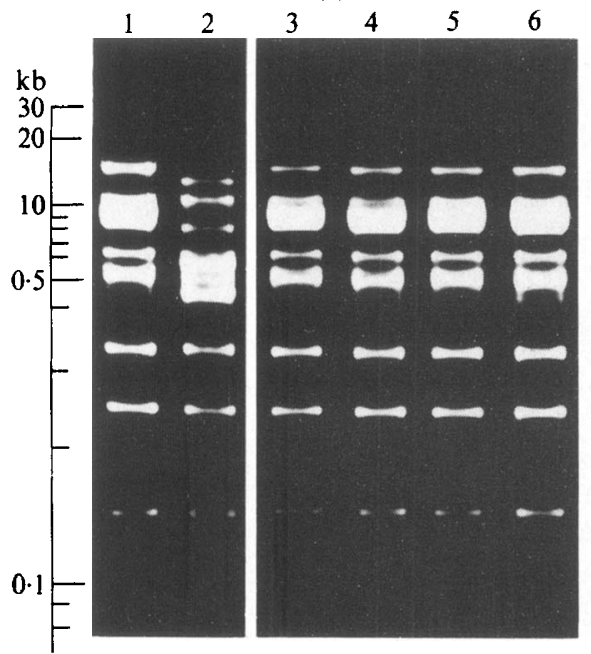

(b)

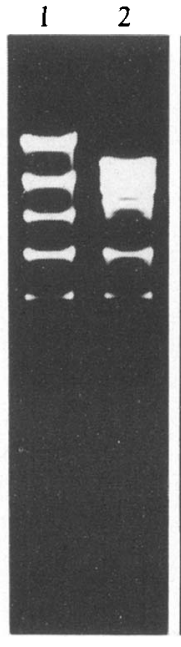

3

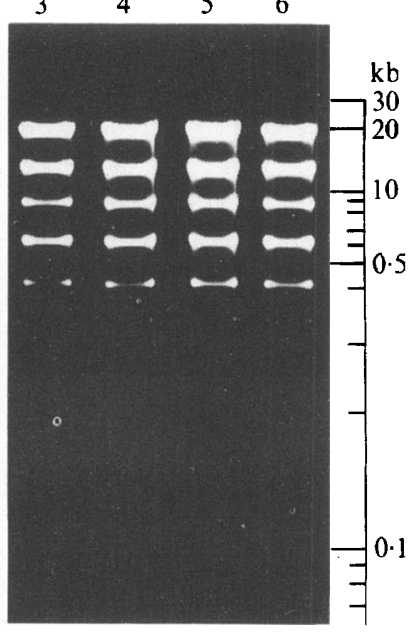

Fig. 2. Uniparental transmission pattern (lanes 3-6) of mtDNA during plasmodium formation by the mating between amoebal strains MB29 (lane 1) and NG9 (lane 2). MtDNAs were digested with Bst NI (a) or XbaI (b). For each of the four plasmodia, the mtDNA digestion patterns were of M-type, the same as MB29.

plasmodia makes it unlikely that plasmodia of different isolates would be able to fuse (Collins, 1972; Collins \& Haskins, 1972).

\section{Uniparental inheritance of $m t D N A$ during plasmodium formation}

The restriction analyses of mtDNA showed that all the plasmodial strains were homoplasmic, i.e. possessing only one type of mtDNA. This suggested that uniparental transmission of mtDNA might occur during plasmodium formation, and the possibility was investigated by mating amoebal strains that carried mtDNA of different types. Amoebal strains MB29 (matA2 matBI; M-type) and NG9 (matA1l matB6; N-type) were isolated as haploid, meiotic progeny of the plasmodial strains $\mathrm{M}_{3} \mathrm{~b}$ and $\mathrm{Ng}$, respectively. Plasmodia were obtained from four independent matings of these amoebal strains and mtDNA from each of the plasmodia was isolated and analysed by restriction endonuclease digestion (Fig. 2). Each plasmodium possessed only one parental type of mtDNA, and in every case it was the M-type. This result demonstrated uniparental inheritance of mtDNA, and suggested a possible bias in favour of one parent (MB29).

Amoebae of $P$. polycephalum are isogametes. It was confirmed by microscopy (unpublished data) that amoebae of strains MB29 and NG9 were approximately equal in size and in content of mitochondria. Thus, it would be expected that amoebae of these strains should contribute roughly equal numbers of mitochondria to heteroplasmic zygotes at the time of fusion. Zygotes develop into multinucleate, syncytial plasmodia by repeated mitotic cycles in the absence of cell division, as well as by fusions among developing plasmodia. Even at the earliest time at which analysis of plasmodia could be done, some 30-40 mitotic cycles after amoebal fusion, there was no evidence that more than one mtDNA type was present. It thus appears that the production of homoplasmic cells from heteroplasmic ones must occur relatively early in the process of vegetative growth and fusion.

A well-documented feature of yeast mitochondrial genetics (reviewed by Birky, 1978; Birky et al., 1982) is that heteroplasmic cells may produce homoplasmic progeny, so that alleles appear to segregate during mitotic as well as meiotic divisions. This vegetative segregation is caused in part by random partitioning of cytoplasmic organelles or DNA molecules between daughter cells at each cell division. Another factor is intracellular drift of gene frequencies, due largely to random sampling of organelle DNA molecules for replication. In plasmodium formation by $P$. 
polycephalum, however, random partitioning of mitochondria or of mtDNA molecules by cell division cannot be a significant factor in vegetative segregation. The biased uniparental transmission of mtDNA suggests that a non-random mechanism may be operating, such as preferential replication of one of the two mtDNA species. To determine the nature of such nonrandom processes, it will be necessary to develop methods to study the precise behaviour of mitochondria and mtDNA molecules during the earliest stages of plasmodial development.

The uniparental inheritance of mtDNA documented in the present work is only one of several phenomena involving vegetative segregations in $P$. polycephalum. For example, plasmodial heterokaryons containing both haploid and diploid nuclei show instability, breaking down to yield homokaryons containing nuclei only of the original haploid component (Dee \& Anderson, 1984); it is thought that this type of instability may be involved in the phenomenon of 'stimulated selfing' (Anderson \& Truitt, 1983), in which the plasmodia arising in a cross are sometimes found to include haploids of parental genotype as well as diploids. Another kind of heterokaryon instability is under the control of incompatibility genes known as let (Carlile, 1976) or kil (Adler \& Holt, 1974). Following plasmodial fusion, nuclei carrying dominant let alleles apparently cause destruction of nuclei carrying recessive alleles of the same let loci (Border \& Carlile, 1974; Lane \& Carlile, 1979). It will be of interest to determine whether the mechanism of uniparental inheritance of mtDNA in any way parallels these examples of uniparental nuclear inheritance.

We greatly thank Drs M. J. Carlile, S. Hatano, E. Kamitsubo, N. Kamiya, Y. Kobatake, K. Kuroda (Osaka University), A. Nishi, J. Ohta (Ochanomizu University), Y. Sakaki (Kyushu University), F. Sawada and D. Pallotta (Laval University) for the gifts of strains. This work was supported in part by Grant no. 60740391 (S.K.) and a research scholarship abroad from the Ministry of Education, Science and Culture of Japan.

\section{REFERENCES}

AdLer, P. N. \& Holt, C. E. (1974). Genetic analysis of the Colonia strain of Physarum polycephalum: strains that mate with and are partially isogenic to the Colonia strain. Genetics 78, 1051-1062.

ANDERSON, R. W. (1976). Analysis of the amoebalplasmodial transition in Physarum polycephalum. $\mathrm{PhD}$ thesis, University of Leicester, UK.

ANDERSON, R. W. (1977). A plasmodial colour mutation in the Myxomycete Physarum polycephalum. Genetical Research 30, 301-306.

ANDERSON, R. W. (1979). Complementation of amoebal-plasmodial transition mutants in Physarum polycephalum. Genetics 91, 409-419.

Anderson, R. W. \& TruitT, C. L. (1983). A new type of plasmodium formation in Physarum polycephalum. Genetical Research 42, 285-296.

Anderson, R. W., Gray, A., Huchins, G. \& Price, J. (1983). Preservation of Physarum polycephalum amoebae with anhydrous silica gel. Physarum Newsletter 15, 3

BIRKY, C. W., JR (1978). Transmission genetics of mitochondria and chloroplasts. Annual Review of Genetics 12, 471-512.

BIRKY, C. W., JR, ACton, A. R., Dietrich, R. \& CARVER, M. (1982). Mitochondrial transmission genetics: replication, recombination, and segregation of mitochondrial DNA and its inheritance in crosses. In Mitochondrial Genetics, pp. 333-348. Edited by G. Attardi, P. Borst \& P. P. Slonimski. Cold Spring Harbor, New York: Cold Spring Harbor Laboratory.
Border, D. J. \& Carlile, M. J. (1974). Somatic incompatibility following plasmodial fusion between strains of the Myxomycete Physarum polycephalum: the effect on their nuclei. Journal of General Microbiology 85, 211-219.

CanN, R. L., Brown, W. M. \& Wilson, A. C. (1984). Polymorphic sites and the mechanism of evolution in human mitochondrial DNA. Genetics 106, 479-499.

Carlile, M. J. (1976). The genetic basis of the incompatibility reaction following plasmodial fusion between two strains of the Myxomycete Physarum polycephalum. Journal of General Microbiology 93, $371-376$.

Castora, F. J., Arnheim, N. \& Simpson, M. V. (1980). Mitochondrial DNA polymorphism: evidence that variants detected by restriction enzymes differ in nucleotide sequence rather than in methylation. Proceedings of the National Academy of Sciences of the United States of America 77, 6415-6419.

Collins, O. R. (1972). Plasmodial fusion in Physarum polycephalum: genetic analysis of a Turtox strain. Mycologia 64, 1130-1137.

Collins, O. R. (1975). Mating types in five isolates of Physarum polycephalum. Mycologia 67, 98-107.

Collins, O. R. \& Haskins, E. F. (1972). Genetics of somatic fusion in Physarum polycephalum: the P PII strain. Genetics 71, 63-71.

COOKE, D. J. (1974). Studies on the Colonia isolate of Physarum polycephalum. Ph.D thesis, University of Leicester, UK.

COOKE, D. J. \& DEE, J. (1975). Methods of the isolation 
and analysis of plasmodial mutants in Physarum polycephalum. Genetical Research 24, 175-187.

DANIEL, J. W. \& BALDWIN, H. H. (1964). Methods of culture for plasmodial Myxomycetes. Methods in Cell Physiology 1, 9-41.

DEE, J. (1960). A mating-type system in an acellular slime-mould. Nature, London 185, 780-781.

DEE, J. (1982). Genetics of Physarum polycephalum. In Cell Biology of Physarum and Didymium, vol. 1, pp. 211-251. Edited by H. C. Aldrich \& J. W. Daniel. New York: Academic Press.

DeE, J. \& ANDERSON, R. W. (1984). The effect of ploidy on the stability of plasmodial heterokaryons in Physarum polycephalum. Journal of General Microbiology 131, 1167-1179.

Ferris, S. D., Brown, W. M., Davidson, W. S. \& WILSON, A. C. (1981). Extensive polymorphism in the mitochondrial DNA of apes. Proceedings of the National Academy of Sciences of United States of America 78, 6319-6323.

Hudspeth, M. E. S., Shumard, D. S., Tatti, K. M. \& Grossman, L. I. (1980). Rapid purification of yeast mitochondrial DNA in high yield. Biochimica et biophysica acta 610, 221-228.

Honey, N. K., Poulter, R. T. M. \& Teale, D. M. (1979). Genetic regulation of differentiation in Physarum polycephalum. Genetical Research 34, 131142.

Kaneko, T., Terachi, T. \& Tsunewaki, K. (1986). Studies on the origin of crop species by restriction endonuclease analysis of organellar DNA. II. Restriction analysis of ctDNA of 11 Prunus species. Japanese Journal of Genetics 61, 157-168.

Kawano, S., Suzuki, T. \& Kurolwa, T. (1982). Structural homogeneity of mitochondrial DNA in the mitochondrial nucleoid of Physarum polycephalum. Biochimica et biophysica acta 696, 290-298.

$$
\text { in we line }
$$

Kawano, S., Nishibayashi, S., Shiraishi, N., MiYaHARA, M. \& KuROIWA, T. (1983). Variance of ploidy in mitochondrial nucleus during spherulation in Physarum polycephalum. Experimental Cell Research 149, 359-373.

Kawano, S., Kuroiwa, T. \& Anderson, R. W. (1987). A third multiallelic mating type locus in Physarum polycephalum. Journal of General Microbiology 133, 2539-2546.
Kirouac-Brunet, J., Masson, S. \& Pallotta, D. (1981). Multiple allelism at the matB locus in Physarum polycephalum. Canadian Journal of Genetics and Cytology 23, 9-16.

Lane, E. B. \& Carlile, M. J. (1979). Post-fusion somatic incompatibility in plasmodia of Physarum polycephalum. Journal of Cell Science 35, 339-353.

MohberG, J. \& BABCOCK, K. L. (1982). Genealogy and characteristics of some cultivated isolates of Physarum polycephalum. In Cell Biology of Physarum and Didymium, vol. 1, pp. 273-283. Edited by H. C. Aldrich \& J. W. Daniel. New York: Academic Press.

SAGER, R. (1972). Cytoplasmic Genes and Organelles. New York: Academic Press.

Sanders, J. P. M., Borst, P. \& WeiJers, J. (1975). The organization of genes in yeast mitochondrial DNA. II. The physical map of Eco RI and HindII + III fragments. Molecular and General Genetics 143, 5364.

Shumard, D. S., Grossman, L. I. \& Hudspeth, M. E. S. (1986). Achlya mitochondrial DNA: gene localization and analysis of inverted repeats. Molecular and General Genetics 202, 16-23.

Turner, G., Earl, A. J. \& Greaves, D. R. (1982). Interspecies variation and recombination of mitochondrial DNA in the Aspergillus nidulans species group and the selection of species-specific sequences by nuclear background. In Mitochondrial Genes, pp. 411-414. Edited by G. Attardi, P. Borst \& P. P. Slonimski. Cold Spring Harbor, New York: Cold Spring Harbor Laboratory.

Wakasugi, M. \& OHTA, J. (1973). Studies on the amoebo-flagellate transformation on Physarum polycephalum. Botanical Magazine (Tokyo) 86, 290-308.

WhEALS, A. E. (1970). A homothallic strain of the Myxomycete Physarum polycephalum. Genetics 66, 623-633.

Youngman, P. J., Pallotta, D. J., Hosler, B., Struhl, G. \& Holt, C. E. (1979). A new mating compatibility locus in Physarum polycephalum. Genetics 91, 683-693.

Youngman, P. J., Anderson, R. W. \& Holt, C. E. (1981). Two multiallelic mating compatibility loci separately regulate zygote formation and zygote differentiation in the Myxomycete Physarum polycephalum. Genetics 97, 513-530. 